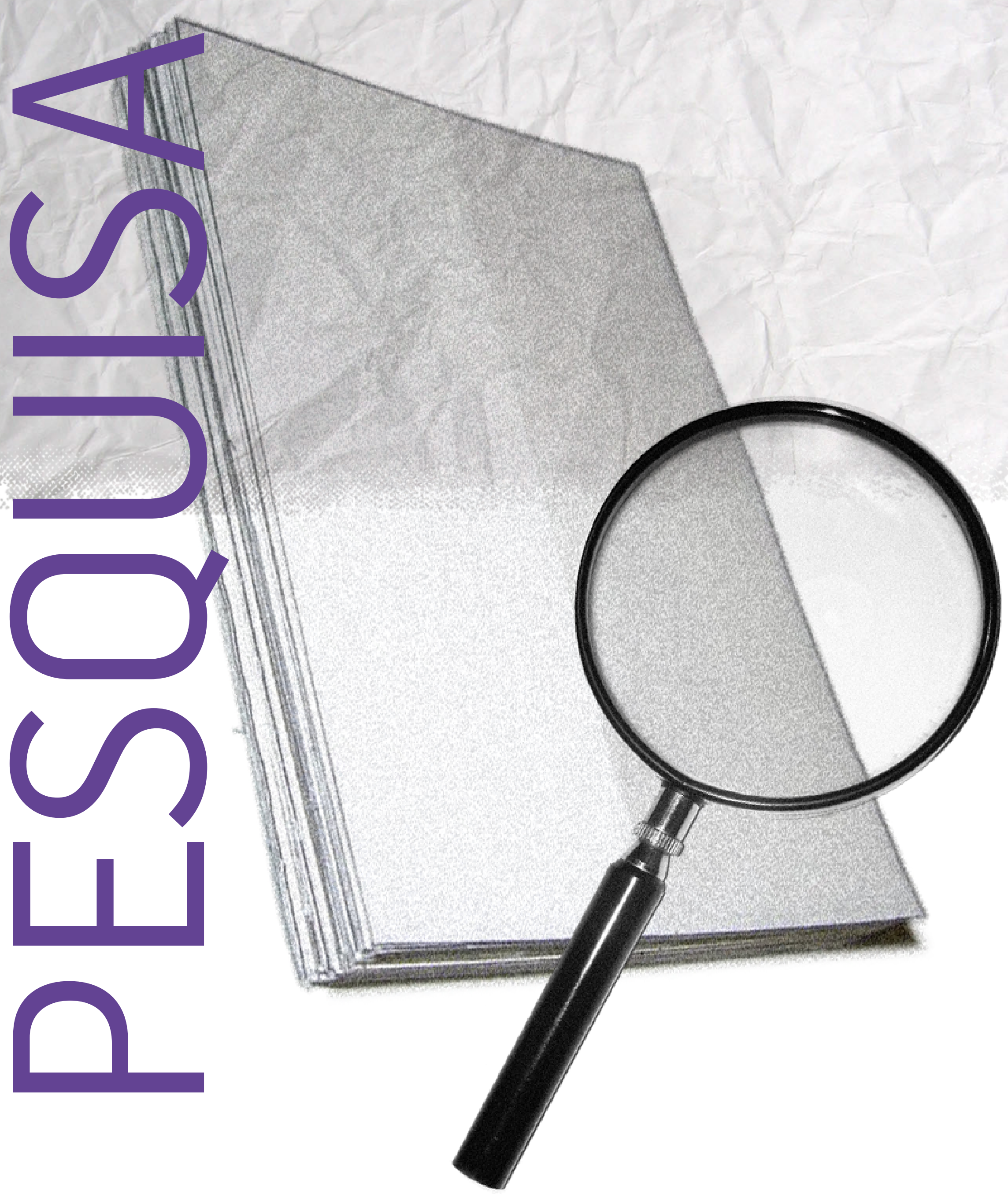




\section{Prácticas comunicativas y perspectivas para el cambio social en las organizaciones no gubernamentales (ONGs), en España y Brasil}

Communication practices and perspectives for the social change in non-governmental organizations (NGOs), in Spain and Brazil

Práticas comunicativas e perspectivas para a mudança social nas organizações não-governamentais (ONGs), na Espanha e no Brasil

\section{Antonio Castillo Esparcia}

- Doctor en Comunicación por la Universidad Autónoma de Barcelona (UAB)

- Profesor de Relaciones Públicas en la Universidad de Málaga (UMA)

- Director de la Maestría en Dirección Estratégica e Innovación en Comunicación y del Doctorado en Gestión Estratégica en Comunicación, de la UMA

- E-mail:acastilloe@uma.es

\section{Margarida Maria Krohling Kunsch}

- Profesora titular de la Escola de Comunicações e Artes de la Universidade de São Paulo (ECA-USP)

- Profesora asociada en Teoría de la Comunicación Institucional: Políticas y Procesos por la ECA-USP

- Doctora y máster en Ciencias de la Comunicación por la ECA-USP

- E-mail:mkkunsch@usp.br

\section{Mariângela Furlan Haswani}

- Doctora por el Programa de Postgrado en Integración de la América Latina, de la Universidade de São Paulo (Prolam-USP)

- Máster en Ciencias de la Comunicación por la Escola de Comunicações e Artes de la Universidade de São Paulo (ECA-USP)

- E-mail:mari.haswani@gmail.com 


\section{ANO 14 • NÚMERO 26 • 1ㅇSM. 2017 • ORGANICOM \\ PRÁCTICAS COMUNICATIVAS Y PERSPECTIVAS PARA EL CAMBIO SOCIAL EN LAS ORGANIZACIONES NO GUBERNAMENTALES (ONGS), EN ESPAÑA Y BRASIL}

\section{Resumen}

Este trabajo examina el papel que la comunicación en las redes sociales digitales desempeña en la consecución de los objetivos de las organizaciones no gubernamentales (ONGs) - u organizaciones del tercer sector -, en Brasil y en España, como estrategia para llegar a ser conocidas, sensibilizar, educar y alcanzar legitimación social y política en el seno de la sociedad. El artículo presenta, inicialmente, un perfil del tercer sector en Brasil y España - similitudes, diferencias y operación de funciones originalmente estatales-, seguido de una explicación general sobre el desarrollo del tercer sector. A continuación, una investigación comparativa entre 24 entidades españolas y 23 brasileras, levanta, a través de cuestionarios dirigidos a los directores de las ONGs, cuánto, cómo y para qué son utilizados los medios digitales. Entre las conclusiones, la investigación constató que la comunicación de la mayoría de las entidades se centra en la difusión de informaciones y de las actividades y en apenas una pequeña parcela se dirige a la construcción de un diálogo digital, sin aprovechar las posibilidades de comprometimiento con los públicos.

\section{PALABRAS CLAVES: COMUNICACIÓN DIGITAL・ESTRUCTURA COMUNICATIVA・TERCERO SECTOR・BRASIL・ESPAÑA}

\section{Abstract}

This paper examines the role played by the digital social networks communication in achieving the goals of the nongovernmental organizations (NGOs) - or third-sector organizations -, in Brazil and in Spain as a strategy to become known, to raise awareness, to educate and to accomplish social and political legitimacy within society. The article starts by presenting a profile of the NGOs in Brazil and Spain - similarities, differences and operation of originally State functions -, followed by an overview about the development of the third sector. Subsequently, a comparative investigation across 24 Spanish and 23 Brazilian entities determines, through questionnaires addressed to the directors of the NGOs, how much, how and what for the digital media are used. Among the conclusions reached, the research has found that the communication of most entities is focused on the diffusion of information and activities and only a small part is addressed to the development of a digital dialogue, not taking advantage of the possibilities of engagement with the audiences.

KEYWORDS: DIGITAL COMMUNICATION •COMMUNICATIVESTRUCTURE・THIRDSECTOR・NON-GOVERNMENTAL ORGANIZATIONS •SPAIN •BRAZIL.

\section{Resumo}

Este trabalho examina o papel que a comunicação nas redes sociais digitais desempenha na consecução dos objetivos das organizações não-governamentais (ONGs) - ou organizações do terceiro setor -, no Brasil e na Espanha, como estratégia para se tornarem conhecidas, sensibilizar, educar e alcançar legitimação social e política no seio da sociedade. 0 artigo apresenta, inicialmente, um perfil das ONGs no Brasil e na Espanh - semelhanças, diferenças e operação de funções originalmente estatais -, seguido de uma explanação geral sobre o desenvolvimento do terceiro setor. Em seguida, uma investigação comparativa entre 24 entidades espanholas e 23 brasileiras apura, por meio de questionários direcionados aos diretores das ONGs, quanto, como e para que são utilizados os meios digitais. Entre as conclusões, a pesquisa constatou que a comunicação da maioria das entidades está centrada na difusão de informações e das atividades e apenas uma pequena parcela é direcionada à construção de um diálogo digital, não aproveitando as possibilidades de engajamento com os públicos. 


\section{ANO 14 • NÚMERO 26 • 1ㅇSM. 2017 • ORGANICOM PRÁCTICAS COMUNICATIVAS Y PERSPECTIVAS PARA EL CAMBIO SOCIAL EN LAS ORGANIZACIONES NO GUBERNAMENTALES (ONGS), EN ESPAÑA Y BRASIL}

asorganizaciones no gubernamentales (ONGs) surgieron de la sociedad civil para intentar paliar situaciones noafrontadas por los poderes públicos. Desde hace algunas décadas, dentro del campo económico, político y social, ofrecen a las poblaciones una posibilidad de desarrollo que estas muy difícilmente conseguirían alcanzar de otra forma. Para lograr su intento, las ONGs necesitan realizar estrategias de comunicación que les permiten darse a conocer, sensibilizar, educar y legitimarse social y políticamente. La presente investigación tuvo como objetivo exactamente conocer qué papel desempeña la comunicación en el contexto del tercer sector, más concretamente en las organizaciones no gubernamentales.

El binomio sociedad y Estado ha sido estudiado desde múltiples relaciones a partir de la creación del Estado moderno en el siglo XVIII. Las diferentes ideologías han puesto el énfasis en alguno de los dos polos y de ahí que en esta investigación se expliquen las funciones y los quehaceres de las organizaciones no gubernamentales. Asimismo, la complejidad social con numerosas demandas sociales ha hecho que las necesidades de la sociedad hacia el Estado se incrementen de manera exponencial, por lo que ha sido necesario que también problemas sociales sean discutidos y tratados desde la propia sociedad al no poder asumir desde el Estado todas las peticiones sociales. Y los órganos que las han presentado en el centro de sus actividades son las organizaciones no gubernamentales, que ayudan a solucionar los problemas sociales.

Con este planteamiento nuestro estudio incide en estas organizaciones y en los flujos comunicativos que generan y proyectan hacia la sociedad y hacia el Estado.

\section{LAS ORGANIZACIONES NO GUBERNAMENTALES EN ESPAÑA Y BRASIL}

Las organizaciones no gubernamentales (ONGs) han sufrido profundas transformaciones en España, debido a la crisis económica, que ha influido en su estructura financiera y, por extensión, en sus estrategias de comunicación. Los valores asociados a las ONGs como elementos dinámicos de participación política (Offe, 1996), con objetivos heterogéneos (Goldstein, 1979) y con ciclos de surgimiento y consolidación (Avina, 1993), las convierten en auténticos actores sociales y políticos que desempeñan funciones que llegan a aquellos ámbitos en los que los poderes públicos se muestran ineficaces.

Las diferentes definiciones de ONGs ponen énfasis en determinadas características como:

- Organizaciones surgidas de la iniciativa civil (González, 2006; Sampedro, Jerez Novara y López Rey, 2002);

•Independencia del poder gubernamental (Marcuello, 2007; Granda y Lama, 1987);

- Que se forman a través de redes sociales de confianza que facilitan la cooperación.

La verdadera explosión de las ONGs en España se produce en los años 1980, como consecuencia de la consolidación de la democracia y de una creciente estructura de la sociedad civil española (Gómez Gil, 2005; González, 2006). Esos primeros pasos también suponen una creciente profesionalización de sus estructuras como consecuencia de la consolidación de los balances económicos.

Hasta mediados de los años 1980 no se produce la generalización de una estructura comunicativa de las ONGs (Gómez Gil, 2005; Sampedro, Jerez Novara y López Rey, 2002; Almansa, 2011). Sin embargo, estas estructuras comunicativas son muy básicas y se centran en las relaciones informativas con los medios de comunicación a través de comunicados y ruedas de prensa. El 
papel de los medios de comunicación es el de ser difusor de algunas de sus actividades, pero la acción no es sistemática ni planificada con una estrategia de comunicación (Castillo, 2007; Almansa, 2011; González, 2006; García Orosa, 2006).

El papel de la comunicación en las ONGs debe encuadrarse en una estrategia de comunicación en la que puedan establecerse relaciones de confianza y diálogo con los diferentes públicos a través de diversas técnicas de comunicación (Almansa, 2011). Todo ello para conseguir una serie de objetivos como son:

- Focalización de las temáticas que defiende la organización;

• Educación sobre la población y los líderes políticos y sociales (Psacharopoulos; Woodhall, 1987);

- Sensibilización social (Ortega, 2007);

- Legitimidad para defender demandas sociales (Sikirit, 2003; Castillo, 2007);

- Transparencia sobre la actuación y la asignación de recursos;

- Reclutamiento de miembros, asociados, simpatizantes y colaboradores (Sikirit, 2003; Seo, Kim y Yang, 2009);

- Fomento de la participación a través de los actores que se convocan o mediante el activismo social o digital.

Los estudios sobre la relación entre ONGs e internet se han centrado en el análisis del engagement entre organización y públicos (Kang y Norton, 2004; Ingehoff y Koelling, 2007; Yang y Taylor, 2010); y en aspectos específicos de la comunicación como organizaciones solidarias. En este sentido, Kang y Norton (2004, p. 279-280) apuntan a la escasa interacción de sus espacios web: "La mayoría de las organizaciones usaba en sus web sites um design simple, con un mínimo de animación (...). Sin embargo, las organizaciones no está utilizando los beneficios de la web para atraer más visitantes".

En Brasil, la nueva Constitución en vigor desde 1988, conocida como "Constitución ciudadana", asegura a los residentes del país derechos fundamentales que están recogidos en los artículos 5 y 6 , pero también hay otros apartados que inciden sobre esos derechos a lo largo del documento. Referente a los derechos sociales, la norma normarum indica educación, salud, alimentación, trabajo, vivienda, transporte y, por extensión, asistencia social, protección de la maternidad y la infancia y asistencia a los desamparados como obligaciones del Estado, por lo que deben estar regulados y se deben realizar acciones que puedan garantizar el acceso universal de los ciudadanos a los derechos citados.

Con el advenimiento de la globalización, la presencia creciente de los gobiernos en los negocios internacionales produjo un redimensionamiento de las operaciones estatales en el ámbito nacional y la consecuente delegación de sus obligaciones sociales al sector privado, de manera específica en las organizaciones del tercer sector, principalmente en la concesión de gestiones. De esta forma, las organizaciones sociales pasan a gestionar grandes áreas de asistencia propias de la responsabilidad social.

En el panorama actual, conforme a una investigación de la Abong - Asociación Brasilera de Organizaciones no Gubernamentales (Abong, 2010) sobre las Fundaciones Privadas y Asociaciones sin Ánimo de Lucro (Fasfil), señalaba las 
áreas de actuación de estas últimas instituciones. Del total, 82,9 mil (28,5\%) administraban directamente servicios o ritos religiosos; 44,9 mil (15,5\%) trabajaban en el área de asociaciones patronales y profesionales; y 42,5 mil (14,6\%), en la defensa de los derechos. Existían, además, 54,1 mil entidades (18,6\%) dedicadas a implementar políticas gubernamentales (salud, educación, asistencia social). En este segmento, los grupos más vulnerables de la población - pobres, niños, adolescentes conflictivos y con necesidades especiales - eran asistidos por 30,4 mil entidades de asistencia social (10,5\%). En educación y salud eran 23,7 mil entidades. Destacaban las entidades de asistencia primaria (4,5 mil) y otros servicios de salud (3,9 mil). Las entidades centradas en el medio ambiente y en la protección de los animales representaban un 0,8\% del total de Fasfil.

\section{EL CONTEXTO CONCEPTUAL DEL TERCERO SECTOR}

Rubem César Fernandes (1995, p. 27) entiende que, debido a la distinción entre mercado (segundo sector) y Estado (primer sector),

puede decirse que el tercer sector está compuesto por organizaciones sin fines de lucro, creadas y mantenidas por el entusiasmo de la participación voluntaria, en al ámbito no gubernamental, en continuidad con las tradicionales prácticas de caridad, filantropía, patrocinios y extendiendo su sentido para otros dominios gracias, principalmente, a la inclusión del concepto de ciudadanía de sus manifestaciones variadas en la sociedad civil.

Mucho más complejo es el abordaje de Lester Salomon (2005, p. 93-99), que describe el concepto del tercer sector en tres perspectivas: como idea, como realidad y como ideología. Como idea, se centra en el campo de los valores, que pueden ser espirituales, religiosos, morales o políticos. Como realidad, aborda el potencial económico del tercer sector, en el contexto de la invisibilidad: "la diversidad del sector es tan asombroso, que nos induce a pasar por alto las considerables similitudes que también existen en él. Las personas ocupadas con el desarrollo no quieren ser confundidas con las que se devotan a la mera asistencia" (Salomon, 2005, p. 93). Como ideología, el autor apunta esta perspectiva como el oportunismo resultante de las indefiniciones de las otras dos perspectivas, mostrando que, "por el acaso o a propósito, el tercer sector pasó a ser usado como una cortina de humo para una serie de preocupaciones políticas e ideológicas conflictivas, que poco tienen a ver con la realidad o la idea del tercer sector" (Salomon, 2005, p. 96).

Con esta visión, Pontes y Bava (1996, p. 134-135) definen al tercer sector a partir de la idea del empoderamiento de la sociedad civil por medio de articulaciones y movilizaciones en el campo de las reivindicaciones de los derechos, pero también de la fiscalización del Estado:

En el lenguaje actual de la ciencia política las ONGs tienen como principal motivo para existir el empowerment de los sectores sociales excluidos o discriminados, privados de sus derechos. Fortalecer actores sociales emergentes significaba hace algunos años ayudar en su organización, sistematizar sus reivindicaciones, establecer los contractos que permitían a los movimientos conquistar el apoyo de otros sectores de la sociedad y el espacio público para sus demandas. Un trabajo que se hacía a partir del enfrentamiento con el Estado autoritario y que generó una cultura entre las ONGs y los movimientos sociales, una comprensión del Estado como un enemigo para desafiar permanentemente.

El tridente Estado, mercado e tercer sector está llamado a trabajar unido, para el beneficio social. Marcos Kisil (2005, p. 137) demuestra, en la Tabla 1, que los tres sectores pueden contribuir, "de acuerdo con algunas variables esenciales", para ese objetivo: 
Cuadro 1: Abordajes combinados para el desarrollo de la comunidad

\begin{tabular}{|l|l|l|l|}
\hline \multicolumn{1}{|c|}{ Variables } & \multicolumn{1}{|c|}{ Gobierno } & \multicolumn{1}{c|}{ Mercado } & \multicolumn{1}{c|}{ Tercero sector } \\
\hline Mecanismo principal & Estructuras democráticas & Interacciones de mercado & Asociaciones voluntarias \\
\hline Toma de decisiones & $\begin{array}{l}\text { Funcionarios elegidos, } \\
\text { administradores }\end{array}$ & $\begin{array}{l}\text { Productores individuales, } \\
\text { consumidores, }\end{array}$ & Líderes y miembros \\
\hline $\begin{array}{l}\text { Guías de comportamiento } \\
\text { Criterios para toma } \\
\text { de decisiones }\end{array}$ & Regulaciones & Precios & Acuerdos \\
\hline Modo de operación & De la cima para abajo & Individualista & Interés de los miembros \\
\hline
\end{tabular}

Fuente: Marcos Kisil (2005).

Aunque contenga conceptos aparentemente dispares, algunos todavía en fase de consolidación, la síntesis de SchererWarren (1999, p. 31) parece representar bien los aspectos más comunes entre investigadores:

Desde el punto de vista formal, ONGs son agrupaciones colectivas con alguna institucionalidad, que se definen como entidades privadas con finalidades públicas y sin fin de lucros, contando con alguna participación voluntaria. (...) En ese universo se incluyen tanto las organizaciones meramente recreativas o de participantes como las actuantes en las políticas públicas y en la politización social.

Cualquier que sea el punto de vista empleado para conceptualizar el tercer sector, constatamos una confluencia de los públicos que precisan interrelacionar el modo de concienciación y de movilización, implícito en su ontología. $Y$ en la complejidad de ese contexto que envuelve a Estado, sociedad y tercer sector, la comunicación aparece como protagonista en el proceso, activando canales indispensables en la movilización y en la ejecución de los proyectos de transparencia de los procedimientos y resultados:

Es una comunicación vinculada a la práctica de movimientos colectivos. Retrata momentos de proceso de "contra"-comunicación en la sociedad civil, en sus formas democratizadas de producir comunicación, sus vehículos, contenidos etc., diferentes 0 alternativos, de la comunicación limitada al ámbito de la estructura dominante de comunicación, o sea, de los grandes medios de comunicación de masas. En este marco, la "nueva" comunicación es un grito antes sofocado de denuncia y reivindicación por transformaciones (Peruzzo, 1995, p. 29).

El "grito sofocado" al que se refiere la autora remite a lo ocurrido en el período de la dictadura militar brasileña de 1964, hasta la apertura política, con la comunicación popular producida principalmente por "activistas, intelectuales e investigadores de izquierda. Estas producciones constituyeron especialmente la prensa alternativa - o "nanica" [enana] - dejando como herederas las prensas popular y partidaria" (Wendhausen, 2003, p. 39). Sin embargo, extrañamente las organizaciones del tercer sector y la prensa alternativa no prosperaron. La supervivencia de las ideas, en el periodo de la dictadura, fue acción 
de las Comunidades Eclesiales de Base (CEBs), de la Iglesia Católica, tanto en los grupos segmentados de los miembros como en la Juventud Estudiantil Católica (Juca). En cada grupo, entre grupos diferentes y entre todos ellos en la sociedad, se desarrolló una comunicación comunitaria de gran eficiencia. Bajo el pretexto de los estudios del Evangelio, miembros de los movimientos sociales se reunían para discutir, de hecho, las propuestas de la teología de la liberación, basadas en Leonardo Boff y con las enseñanzas de Paulo Freire en la educación libertaria y crítica.

En este sentido, debemos observar que

las investigaciones sobre comunicación popular necesitan que la teoría abarque los procesos en su contexto más amplio en el que se realiza, o sea, deben ir más allá del estudio del medio comunicativo en sí mismo, de un periódico, por ejemplo, ya que la dinámica social en el que este se halla insertado es lo que le dará significado (Peruzzo, 1998, p. 114).

Históricamente, los medios de comunicación de masas de Brasil restringen su ámbito de a los intereses de sectores económicos y políticos dominantes, soslayando en sus agendas a casi totalidad de los movimientos populares. Así, la dinámica social de los medios, a los cuales se refiere Peruzzo, no abordan la naturaleza de las finalidades del tercer sector, salvo algunas iniciativas aisladas de campañas anuales de televisión, como "Teleton" (SBT - Sistema Brasilero de Televisión) y "Criança esperança" (TV Globo), y algunos programas periodísticos semanales especializados en ONGs.

Participación consciente implica, de acuerdo con Haswani (2013), un proceso que se inicia con la información y se desarrolla en etapas sucesivas, en donde cada una de ellas depende de las anteriores para su aplicación: comunicación (que presupone la existencia de información y una respuesta del receptor), relación (constituida por la comunicación combinada con una mayor permanencia), diálogo o debate (que, además del relacionamiento, permite sintonizar y armonizar puntos de vista conflictivos), participación (en la que todos los actores tienen voz en las discusiones de temas de interés público, aunque sin deliberación).

Ese pensamiento converge con los criterios básicos de movilización social para la comunicación propuestos por Toro (2005, p. 91). El autor entiende que volver colectiva una propuesta de cambio es uno de los grandes desafíos que políticos, gestores públicos y líderes democráticos enfrentan, una vez que las transformaciones sólo son posibles con la participación: "Una convocación de voluntades de los actores implicados, es decir, de las personas que pueden convertir en acciones y decisiones cotidianas los procesos y las conquistas de las que una reforma necesita". Él evoca, aún, la necesaria convergencia de "intereses (política), la convocación de voluntades (erótica, deseo) y nuevas formas de comprender y organizar la realidad (teoría)". Y sentencía que no basta que un programa o un proyecto esté bien planeado y financiado, siendo necesario que sea bien comunicado para movilizar "voluntades y deseos".

También es de Toro la elaboración de trece tesis que abordan el papel de la comunicación de masas en la sociedad, todas indicando la dirección de los cánones de la ciudanía para el uso de ese medio. La primera tesis - la comunicación es un fundamento de la cultura democrática - se describe de la siguiente manera:

Si definimos la democracia como la auto-creación de un sistema en el cual los diferentes personajes e instituciones pueden hacer competir sus intereses en igualdad de condiciones, el enfoque general de la comunicación para la participación democrática debe orientarse para que los diferentes sectores hagan competir sus intereses, sus mensajes y sus símbolos con oportunidades iguales dentro del universo de intereses, mensajes y símbolos que tramita en la sociedad (Toro, 2005, p. 84). 


\section{ANO 14 • NÚMERO 26 • 1ㅇSM. 2017 • ORGANICOM PRÁCTICAS COMUNICATIVAS Y PERSPECTIVAS PARA EL CAMBIO SOCIAL EN LAS ORGANIZACIONES NO GUBERNAMENTALES (ONGS), EN ESPAÑA Y BRASIL}

organizados, los medios digitales aparecen como una salida posible. Son tecnologías de bajo costo de implantación y mantenimiento y de operaciones simples y accesibles. Por lo tanto, son medios aplicables a la realidad brasileña de las organizaciones no gubernamentales.

En esa perspectiva, De Felice (2012, p.147) analiza las transformaciones socioculturales resultantes de las tecnologías interactivas de información, principalmente la pérdida de "la centralidad del papel del informante". El autor insiste en sus estudios que, "a medida que las tecnologías de la comunicación fueron surgiendo, el mensaje pasó a ser transferido por diferentes soportes cada vez más rápidos y cada vez más autónomos, esto es, menos dependientes de la acción y la actividad humana" (De Felice, 2012, p. 149). Además de eso, las narrativas dominantes, que el autor llama de "centrales", ganan seguidores poderosos en el tejido social: ahora son millones de puntos de vista de los emisores en las redes digitales, capaces de desbancar la hegemonía de los medios de masa dando voz y espacio a los movimientos sociales.

Esta característica de inclusión y participación generalizada debe ser entendida como uno de los espíritus fundadores de la acción comunicativa, cuyos rastros podemos encontrar desde las primeras prácticas. Hoy, las arquitecturas digitales de comunicación en red prometen realizarlas tecnológicamente, mediante el acceso universal a todas las informaciones y la implementación del derecho de todos (individuos, empresas e instituciones) al mismo poder comunicativo, independientemente de la posición geográfica o social (De Felice, 2012, p. 152).

Esteves (2012, p. 219) avanza el pensamiento de De Felice y desafía a las instituciones:

En el contexto de las nuevas tecnologías de la información y la comunicación (NTIC), cabe a las organizaciones sociales en general reajustar profundamente las cuestiones de ciudadanía en el ámbito de su funcionamiento - y, mui especialmente, en el contexto de sus prácticas y procesos comunicacionales. El desafío planteado pasa por asumir una posición ofensiva frente a los cambios: la movilización y la participación en términos deliberativos de las organizaciones en los procesos sociales globales (en oposición a su alineamiento con las lógicas de la colonización corporativa y del control administrativo).

Se trata de hecho de la democratización del acceso, de la voz y quizás de las decisiones, ya que de acuerdo a Joshua Cohen (1997, p. 12), "la concepción de democracia deliberativa está organizada en torno a un ideal de justificación política, según el cual justificar el ejercicio del poder político colectivo es proceder basado en la argumentación pública libre entre iguales; una democracia deliberativa institucionaliza este ideal".

¿Cuál debe ser el papel de la comunicación para desarrollary fortalecer una mejor ciudadanía y una democracia participativa? ¿Qué medios e instrumentos de comunicación se deben utilizar? ¿Qué medios e instrumentos de comunicación son más utilizados? ¿Cómo las tecnologías interactivas de información y los medios digitales sirven de soporte y contribuyen a que las organizaciones no gubernamentales (ONGs) puedan contribuir a sus fines de actuación y se produzcan relaciones con sus públicos? Para tener una percepción del grado de participación y de los instrumentos más utilizados en las prácticas comunicativas más corrientes en las ONGs de España y Brasil, fue realizada una investigación empírica en una muestra representativa de estas organizaciones en los dos países.

\section{MATERIAL Y MÉTODOS}

La investigación tuvo como objetivo principal investigar y analizar las prácticas comunicativas de las ONGs y verificar cómo la comunicación puede contribuir a su misión y los medios más utilizados de acuerdo con sus objetivos organizativos. 
El método adoptado para la investigación empírica ha sido el método cuantitativo. La investigación cuantitativa tiene como objetivo medir, contar y establecer relaciones. En este estudio se adoptó para la investigación un banco de datos que pudiera extraer una muestra de ONGs que sean las más representativas del sector.

\section{Universo y muestra}

El universo se ha compuesto de una base de datos, por medio de la investigación de las principales ONGs España y de Brasil. Para ello se recurrió a la Associação Brasileira de Organizações Não-Governamentais - Abong) y a otros posibles registros institucionales. Por el lado de España, se han analizado las principales ONGs integradas en la Coordinadora de ONG para el Desarrollo (Congde).

En este sentido, el criterio adoptado ha sido consultar los espacios webs oficiales de la Abong y la Congde, para localizar un conjunto de organizaciones que de alguna manera se integrasen en los objetivos del artículo y, por lo tanto, fuera posible analizar como estas entidades han contribuido en las transformaciones sociales y cómo está estructurada su comunicación. Así, fueron seleccionadas las ONGs filiadas à Abong a partir de los siguientes temas: agricultura; medio ambiente; cuestión indígena; cuestión agraria; cuestión urbana; comunicación; organización popular; fortalecimiento de otras ONGs y movimientos sociales; e educación. Con estos temas se han seleccionado un total de 218 ONGs de Brasil. En el caso de España, se han seleccionado las 24 ONGs que disponen de mayor presupuesto en la Congde.

De estas 218 organizaciones de Brasil y las 24 de España se realizó una búsqueda en sus sites, para reunir datos sobre identificación, dirección, organización, pero también para verificar su estructura de comunicación, los medios utilizados, la presencia en redes sociales. Otros ítems se han extraído de los datos suministrados en los cuestionarios remitidos. En el caso de las ONGs españolas el instrumento han sido cuestionarios y análisis de sus espacios web.

\section{Técnicas de recolección de datos}

La principal técnica para la aplicación ha sido establecer un contacto directo con las 218 ONGs de Brasil, por medio del correo electrónico, mediante el envío de una carta explicando el objeto de estudio, conjuntamente con el cuestionario. Se ha obtenido un retorno de 23 respuestas, después de tres tentativas, ya que no existe una cultura por parte de las organizaciones para facilitar y atender demandas de investigaciones académicas. En el caso de España, todas las 24 ONGs han respondido al cuestionario.

\section{ANÁLISIS Y RESULTADOS}

De las respuestas obtenidas se describen a seguir los resultados en relación a las temáticas que integraban el cuestionario.

\section{Datos generales}

\section{Fecha de creación}

En el caso de la fecha de creación de las ONGs analizadas, el resultado es consecuencia de la propia evolución social en la que desarrollan su actividad. Así, las ONGs son entidades que surgen de la iniciativa social y su génesis está 
imbricada con el grado de estructuración social, por lo que podemos decir que la complejidad social implica un mayor número de asociaciones no gubernamentales.

En los dos países analizados los años 1980 se configuran como la década en la que mayor creación de ONGs se ha producido, suponiendo el 34,7\% en Brasil y el 45,8\% en España. En segundo lugar, los años 1990 son los de mayor surgimiento de ONGs, con el $30,4 \%$ en Brasil y el 20,8\% en España. Por lo tanto podemos señalar que son asociaciones que han surgido cuando la sociedad civil ha ido adquiriendo su propia personalidad. En el caso de España es fruto del asentamiento asociativo tras la instauración de la democracia.

Tabla 1: Año de creación por décadas.

\begin{tabular}{|c|c|c|c|c|}
\hline Año & Cantidad (Brasil) & $\%$ & Cantidade (España) & $\%$ \\
\hline 1960 & 1 & 4,3 & 4 & 16,6 \\
\hline 1970 & 3 & 13,0 & 3 & 12,5 \\
\hline 1980 & 8 & 34,7 & 5 & 45,8 \\
\hline 1990 & 7 & 30,4 & 1 & 4,8 \\
\hline 2000 & 4 & 17,3 & 24 & --- \\
\hline Total & 23 & ---11 & 4 \\
\hline
\end{tabular}

Fuente: elaboración propia.

\section{Ramo de temática de actuación}

Las temáticas y los intereses defendidos son consecuencia de la propia gestación de una ONG, puesto que surgen a partir de necesidades sociales que no están satisfechas, en parte o en su totalidad, por los poderes públicos. En el caso de Brasil, derechos humanos y medio ambiente han sido las principales actividades a las que se dedican las ONGs, mientras que en España derechos humanos y ciudadanía han sido los temas preponderantes. En el caso español el primero de los temas (derechos humanos) es una actividad realizada en la esfera internacional y la segunda temática (ciudadanía) está relacionada con la defensa de colectivos de género y de orientación sexual desde una perspectiva de demanda de legislación.

Tabla 2: Ramo de temática de actuación.

\begin{tabular}{|l|c|c|c|c|}
\hline Temática & Cantidad (Brasil) & $\%$ & Cantidade (España) & $\%$ \\
\hline Medio ambiente y educación socioambiental & 4 & 17,3 & 4 & 16,6 \\
\hline Educación & 2 & 8,6 & 3 & 12,5 \\
\hline
\end{tabular}


ANO 14 • NÚMERO 26 • 1은. 2017 - ORGANICOM PRÁCTICAS COMUNICATIVAS Y PERSPECTIVAS PARA EL CAMBIO SOCIAL EN LAS ORGANIZACIONES NO GUBERNAMENTALES (ONGS),

EN ESPAÑA Y BRASIL

\begin{tabular}{|c|c|c|c|c|}
\hline Temática & Cantidad (Brasil) & $\%$ & Cantidade (España) & $\%$ \\
\hline Ciudadanía - Derechos civiles - sociedad civil & 6 & 26,0 & 6 & 25,0 \\
\hline Derechos humanos & 4 & 17,3 & 11 & 45,8 \\
\hline Derecho de las mujeres - feminismo & 3 & 13,0 & --- & --- \\
\hline $\begin{array}{l}\text { Agroecologia y educación popular - sectores } \\
\text { y organizaciones populares públicos }\end{array}$ & 3 & 13,0 & --- & --- \\
\hline $\begin{array}{l}\text { Población indígena - preservación } \\
\text { y conservación }\end{array}$ & 1 & 4,3 & --- & --- \\
\hline Total & 23 & --- & 24 & --- \\
\hline
\end{tabular}

Fuente: elaboración propia.

\section{Financiación}

El elemento económico ha sido uno de los grandes motivos de discusión de las ONGs, puesto que la independencia económica permite una mayor libertad en las actividades y las acciones que se puedan desarrollar. Desde la perspectiva teórica, una ONG posee un marcado carácter de iniciativa privada que cuenta con la participación de socios en la financiación. Sin embargo, de manera progresiva el Estado ha ido generando ámbitos presupuestarios que han ido integrándose en el balance económico de las ONGs. Esa actividad genera discusión sobre el grado de independencia de las ONGs a partir del grado de distribución pública/privada de cada organización. La financiación pública puede ser finalista cuando condiciona la asignación de recursos económicos a la realización de determinadas actividades en determinados espacios prioritarios para el país. Por otro lado, también la financiación puede ser genérica en el momento en el que se hace una asignación de recursos sin ningún tipo de condicionamiento. Es el caso, por ejemplo, de la posibilidad que tienen las personas de, en su declaración de ajuste anual, destinar una parte del impuesto debido a la financiación de entidades sociales.

Las condiciones económicas generales condicionan sobremanera la capacidad estatal de destinar recursos a las asociaciones sociales. Así la crisis económica ha motivado una disminución de los presupuestos públicos, con la consiguiente pérdida de recursos económicos en un momento en el que las necesidades de ayuda y asistencia se hacen más necesarias. Es la situación en la que se encuentran las ONGs cuando deben mantener sus programas de actuación en unas circunstancias de reducción de recursos económicos. A su vez la crisis hace que el número de socios disminuya, así como la cantidad dedicada por las personas.

Todo este panorama ha hecho que las ONGs deben potenciar sus acciones de captación de recursos económicos (fundraising), ya sea de personas individuales como de personas jurídicas. $Y$ en esa nueva estrategia la comunicación desempeña un rol esencial, puesto que conecta las necesidades, las actividades y los ámbitos con los posibles donantes. Consecuencia de ello es que las ONGs han visto como el binomio financiación pública/privada se focalizaba hacia lo privado. 


\section{Tabla 3: Financiación pública/privada}

\begin{tabular}{|l|c|c|c|c|c|c|c|c|}
\hline \multirow{2}{*}{ Financiación } & \multicolumn{4}{|c|}{ Pública } & \multicolumn{4}{c|}{ Privada } \\
\cline { 2 - 11 } & \multicolumn{2}{|c|}{ Brasil } & \multicolumn{2}{c|}{ España } & \multicolumn{3}{c|}{ Brasil } & \multicolumn{2}{c|}{ España } \\
\hline Entre 9 e 20\% & 3 & 13,0 & 3 & 12,5 & --- & -- & -- & --- \\
\hline De 21 a 30\% & 5 & 21,7 & 2 & 8,3 & 4 & 17,3 & 12 & 50,0 \\
\hline De 31 a 60\% & 5 & 21,7 & 8 & 33,3 & 5 & 21,7 & 6 & 25,0 \\
\hline De 70 a 90\% & 5 & 21,7 & 11 & 45,8 & 8 & 34,7 & 6 & 25,0 \\
\hline 100\% & 1 & 4,3 & --- & --- & 4 & 17,3 & -- & --- \\
\hline Ninguna & 3 & 13,0 & --- & --- & 1 & 4,3 & --- & --- \\
\hline No responde & 1 & 4,3 & --- & --- & 1 & 4,3 & -- & --- \\
\hline Total & 23 & --- & 24 & --- & 23 & -- & 24 & --- \\
\hline
\end{tabular}

Fuente: elaboración propia.

Así, tal como se observa, las diferencias entre las ONGs españolas y brasileñas han sido relevantes. En los dos países el peso de la financiación pública ha sido superior a la financiación privada. En el caso español, lo que predominó fue una financiación de tipo público que es superior al $70 \%$ en el $45 \%$ de las ONGs analizadas, mientras que en caso de Brasil ese porcentaje de $70 \%$ publico sólo se produjo en el 21,7\% de las ONGs. Consecuentemente el peso de la financiación privada ha sido reducido, siendo en un $50 \%$ de las ONGs españolas inferior al $30 \%$ de su presupuesto total.

Con este panorama general de las ONGs españolas y brasileñas se constatan algunas semejanzas en la década de creación, pero diferencias en el sistema de distribución de la financiación.

\section{Estructura de comunicación}

El papel de la comunicación en la consecución de los objetivos organizativos de las ONGs es relevante, puesto que permite difundir las actividades de la entidad, permite sensibilizar y educar sobre determinadas temáticas y les aporta visibilidad social, que les permite incrementar sus ámbitos de legitimidad social y política para defender sus objetivos organizativos. Es necesario que los responsables de estas organizaciones interioricen las ventajas que aporta la comunicación a la consecución de los objetivos.

\section{Existencia de una estructura}

Por lo que respecta al papel que la comunicación desempeña en las ONGs analizadas, la primera diferencia sustancial fue que en el caso español es una decisión estratégica, puesto que el 100\% de las ONGs poseen una estructura comunicativa, mientras que sólo el 65,2\% de las ONGs brasileñas poseen una estructura comunicativa. 
Tabla 5: Las ONGs tienen una estructura de comunicación?

\begin{tabular}{|l|c|c|}
\hline \multicolumn{1}{|c|}{ Tiene? } & Brasil & España \\
\hline Sí & 15 & 23 \\
\hline No & 6 & -- \\
\hline No responde & 2 & 1 \\
\hline Total & 23 & 24 \\
\hline
\end{tabular}

Fuente: elaboración propia.

\section{Tamaño de la estructura}

Si se analiza el tamaño de la estructura comunicativa a partir del número de personas que en ella trabajan, encontramos bastantes similitudes entre los dos países, puesto que la mayor parte de las ONGs poseía entre 1 y 5 trabajadores en su estructura comunicativa. Sí que es cierto que si comparamos esa cifra con la categoría superior (más de 5 trabajadores), las ONGs brasileñas obtuvieron una mayor frecuencia, por lo que podemos inferir que existen ONGs brasileñas que le otorgan una gran importancia a la comunicación al destinar un número considerable de personas.

Tabla 6: Número de personas que trabajan en la estructura de comunicación, en las ONGs que tienen una.

\begin{tabular}{|l|c|c|c|c|}
\hline \multirow{2}{*}{ Trabajadores } & \multicolumn{2}{|c|}{ Cantidad (Brasil) } & \multicolumn{2}{c|}{ Cantidad (España) } \\
\cline { 2 - 5 } & $\mathrm{N}$. & $\%$ & $\mathrm{~N}$. & $\%$ \\
\hline De 1 a 5 & 11 & 64,7 & 18 & 81 \\
\hline De 6 a 12 & 4 & 23,5 & 4 & 17,3 \\
\hline No responde & 2 & --- & 1 & $\cdots$ \\
\hline Total & 17 & --- & 23 & $\cdots$ \\
\hline
\end{tabular}

Fuente: elaboración propia.

Con relación al tipo de trabajador existen también en la estructura comunicativa colaboradores del departamento de comunicación que no se integran como trabajadores con remuneración, pero que también realizan labores de acción comunicativa. Y en estos tipos de figura se están potenciando mucho las colaboraciones en campañas en redes sociales y en entornos digitales con la figura de los ciberactivistas o cibervoluntarios como personas que dedican parte de su tiempo de ocio a coadyuvar a la difusión de las campañas comunicativas de las ONGs en las redes sociales.

\section{Fecha de creación de la estructura}

Respecto de la fecha de creación de esa estructura comunicativa, hemos encontrado que las frecuencias serán diferentes en las ONGs españolas y brasileñas. En el caso de las españolas, se encuentran mayoritariamente en la década de los años 1990 , mientras que en caso de las brasileñas se han creado esencialmente a partir del año 2000. Sin embargo, esas diferencias no tienen por que ser relevantes, puesto que la creación de las ONGs condiciona la fecha de creación de la estructura comunicativa. Por eso es relevante cruzar los datos de fecha de nacimiento de las ONGs con la fecha de creación de la estructura comunicativa. 
Tabla 7: Año de creación de la estructura de comunicación.

\begin{tabular}{|l|c|c|c|c|}
\hline \multirow{2}{*}{ Período } & \multicolumn{2}{|c|}{ Cantidad (Brasil) } & \multicolumn{2}{c|}{ Cantidad (España) } \\
\cline { 2 - 5 } & $\mathrm{N}$. & $\%$ & $\mathrm{~N}$. & $\%$ \\
\hline Década de 1990 & 3 & --- & 15 & 62,5 \\
\hline De 2000 a 2013 & 11 & 47,8 & 8 & $\cdots$ \\
\hline No informa & 3 & --- & 1 & $\cdots$ \\
\hline $\begin{array}{l}\text { Responde que no posee } \\
\text { estructura de comunicación }\end{array}$ & 6 & --- & --- \\
\hline Total & 23 & --- & 24 & $\cdots$ \\
\hline
\end{tabular}

Fuente: elaboración propia.

En los dos países las franjas cronológicas de mayor creación de ONGs son las mismas, esto es, los años 1980, mientras que las franjas cronológicas de creación de la estructura comunicativa son diferentes, siendo a partir del año 2000 en el caso de Brasil y a partir de 1980 en el caso español. La consecuencia es clara: las ONGs brasileñas nacen sin estructura comunicativa, puesto que no se crean hasta pasados unos veinte años, mientras que en el caso de España la inserción es inmediata y nacen con la estructura de comunicación.

Tabla 7.1: Creación de las ONGs y de la estructura de comunicación.

\begin{tabular}{|l|c|c|c|c|}
\hline \multirow{2}{*}{ Fechas } & \multicolumn{2}{|c|}{ Brasil } & \multicolumn{2}{c|}{ España } \\
\cline { 2 - 5 } & Año & $\%$ & $\%$ & $\%$ \\
\hline $\begin{array}{l}\text { Creación de la mayoría } \\
\text { de las ONGs }\end{array}$ & 1980 & 34,7 & 1980 & 45,8 \\
\hline $\begin{array}{l}\text { Fechas de creación de la } \\
\text { estructura de comunicación }\end{array}$ & $2000-2013$ & 47,8 & $1980-1990$ & 62,5 \\
\hline
\end{tabular}

\section{Principales medios e instrumentos de comunicación}

Por lo que respecta a los principales instrumentos de comunicación, en los dos países las ONGs poseen espacios web como consecuencia del tipo de sociedad digital en el que convivimos. Sin embargo, observamos en el caso español una mayor actividad dirigida a los medios de comunicación, ya que la totalidad de las ONGs analizadas posee acciones dirigidas a los medios de comunicación, mientras que esas acciones periodísticas solo se producen en el $50 \%$ de las ONGs brasileñas. Las razones pueden ser diversas, ya sea por la estructura de sistemas de medios de comunicación, por la preparación del personal que trabaja en la estructura comunicativa, o por diferencia en la creación de esa estructura y el tipo de panorama comunicativo. En este sentido, cuando se creó la estructura comunicativa de las ONGs españolas (años 1980), el casi exclusivo de comunicación eran los medios de comunicación, mientras en el caso de las ONGs brasileñas que crearon su estructura a partir del año 2000 el panorama comunicativo con internet era más diverso. 
Por lo que respecta a otras estrategias de comunicación, como pueden ser campañas de educación o de sensibilización, la dedicación es semejante en el caso de las ONGs españolas y brasileñas.

Tabla 8: Medios e instrumentos de comunicación

\begin{tabular}{|l|c|c|}
\hline \multicolumn{1}{|c|}{ Medios } & Cantidad (Brasil) & Cantidad (España) \\
\hline Site o portal institucional & 20 & 24 \\
\hline Boletines de noticias & 10 & 18 \\
\hline Press releases para prensa & 11 & 23 \\
\hline Entrevistas colectivas & 3 & 4 \\
\hline Actividades de sensibilización & 8 & 16 \\
\hline Campañas educativas & 9 & 5 \\
\hline Otros & 12 & 0 \\
\hline No responde & 2 & 5 \\
\hline
\end{tabular}

Fuente: elaboración propia.

\section{Presencia en redes sociales}

\section{Si están presentes en las redes}

La adaptación a la sociedad digital de las ONGs analizadas ha sido total, puesto que prácticamente todas poseían presencia en las redes sociales. La principal red utilizada es Facebook, ya que prácticamente el 100\% poseen perfiles. Twitter se configura como la segunda opción, aunque ahora ya con diferencias entre Brasil y España. Así, mientras que las ONGs españolas todas poseían presencia en Twitter, solo el 50\% de las ONGs brasileñas tenían actividad. Por el contrario, las ONGs brasileñas se decantaron más que las españolas por Instagram. Sin duda, que la opción de poseer perfiles en unas u otras redes sociales está muy condicionada por la propia estructura de audiencias de redes sociales en cada uno de los países.

Tabla 9: Presencia en redes sociales.

\begin{tabular}{|c|c|c|}
\hline Presencia en & Cantidad (Brasil) & Cantidad (España) \\
\hline Facebook & 20 & 24 \\
\hline Twitter & 12 & 24 \\
\hline Instagram & 6 & 0 \\
\hline No responde & 1 & 0 \\
\hline
\end{tabular}




\section{Persona especializada para la gestión}

Preguntadas las ONGs por la necesidad de disponer de persona especializada y específica para la gestión de las redes sociales, hemos visto que la adaptación de las ONGs españolas es mayor que en las brasileñas. Así, el $90 \%$ de las ONGs españolas tienen un responsable de gestionar las comunidades virtuales (community manager), mientras que poco más del $50 \%$ de las ONGs brasileñas tienen esa figura.

Tabla 10: ¿Cuentan con un responsable específico para gestión de la comunicación 2.0?

\begin{tabular}{|c|c|c|}
\hline ¿Cuentan? & Cantidad (Brasil) & Cantidad (España) \\
\hline Sí & 13 & 21 \\
\hline No & 9 & 1 \\
\hline No responde & 1 & 2 \\
\hline Total & 23 & 24 \\
\hline
\end{tabular}

Fuente: elaboración propia.

\section{Preparación del personal}

Elgrado de preparación del personal del departamento de comunicación muestraque la educación universitaria en comunicación en mayoritaria. Y, dentro de los estudios de comunicación, la mayor frecuencia se produce en el ámbito del periodismo, ya que suponen prácticamente lo mismo en los dos países (42\%), seguidos de los estudios de publicidad y relaciones públicas.

Tabla 11: Formación académica de los que responden que sí.

\begin{tabular}{|c|c|c|c|c|}
\hline \multirow{2}{*}{ Área } & \multicolumn{2}{|c|}{ Cantidad (Brasil) } & \multicolumn{2}{|c|}{ Cantidad (España) } \\
\hline & $\mathrm{N}$. & $\%$ & $\mathrm{~N}$. & $\%$ \\
\hline Periodismo & 7 & 41,1 & 10 & 43,4 \\
\hline Relaciones públicas & 2 & --- & 8 & --- \\
\hline Publicidad y Propaganda & 2 & --- & & --- \\
\hline Ciencias sociales & 1 & --- & 2 & --- \\
\hline Letras & 1 & --- & 1 & --- \\
\hline Sin respuesta & 4 & --- & 2 & --- \\
\hline
\end{tabular}




\section{Actualización de los contenidos}

El hecho de disponer de personal que se dedique a la gestión de las redes sociales implica que la actividad es permanente. Así, las ONGs analizadas mantienen una actualización casi constante de su presencia en redes sociales, ya sea diariamente o semanalmente. Sin duda que son datos generales a las diferentes redes sociales, ya que algunas demandan una presencia diaria (Twitter) por el tipo de características en el modo de comunicación. Eso supone que la adaptación al nuevo entorno digital se ha producido de manera correcta, al menos desde la perspectiva de la producción de mensajes.

Tabla 12: Frecuencia de actualización de los contenidos de las redes sociales.

\begin{tabular}{|l|c|c|}
\hline \multicolumn{1}{|c|}{ Frecuencia } & Cantidad (Brasil) & Cantidad (España) \\
\hline Diariamente & 10 & 18 \\
\hline Semanalmente & 5 & 1 \\
\hline Mensualmente & 1 & 5 \\
\hline Cuando es necesario & 5 & -- \\
\hline Otra & 3 & -- \\
\hline No responde & 4 & - \\
\hline
\end{tabular}

Fuente: elaboración propia.

\section{Objetivos de la comunicación 2.0}

Ahora bien, se analizamos qué están diciendo las ONGs en su presencia en las redes sociales, los resultados nos muestran un marcado carácter unidireccional frente al bidireccional. Para las ONGs españolas y brasileñas, las redes sociales son expositores, difusores de las actividades realizadas, ya que la mayoría de ellas se dedica a dar información general, a difundir sus actividades o a la visibilidad institucional, que son acciones propias de una comunicación unidireccional. Por el contrario, las acciones bidireccionales encaminadas a la construcción de diálogos (engagement) con los públicos implicados con las ONGs poseen un marcado carácter marginal, ya que menos del 10\% de la actividades en las ONGs están enfocadas a la construcción de ese diálogo digital. Eso supone que la adaptación de las estrategias de comunicación no contempla todavía el espacio digital y a las mejores que eso supone para las organizaciones en términos de cercanía, relación, diálogo, empatía e interacción.

Tabla 12: Objetivos de la comunicación 2.0.

\begin{tabular}{|c|c|c|}
\hline Objetivos & Cantidad (Brasil) & Cantidad (España) \\
\hline Prestar informaciones en general & 14 & 23 \\
\hline $\begin{array}{c}\text { Establecer una información directa } \\
\text { con los públicos de interés }\end{array}$ & 17 & 18 \\
\hline
\end{tabular}




\begin{tabular}{|c|c|c|}
\hline Objetivos & Cantidad (Brasil) & Cantidad (España) \\
\hline Dar visibilidad institucional & 16 & 3 \\
\hline Establecer relaciones con los públicos & 1 & 3 \\
\hline Otros & 1 & 1 \\
\hline No responde & 1 & \\
\hline
\end{tabular}

Fuente: elaboración propia.

\section{CONSIDERACIONES FINALES}

LasONGs surgen cuando existenámbitos sociales a los que los poderes públicos no pueden llegar o por razones de eficiencia y eficacia en la gestión de situaciones sociales. Con la extensión del denominado Estado social y democrático de derecho, las instituciones públicas han adquirido la obligación de paliar desajustes sociales, económicos o políticos hacia los más desfavorecidos. En este contexto, tanto las ONGs de Brasil como de España surgen en la década de los años 1980 y se concretan cuando la propia sociedad civil se desarrolla y fortalece en organizaciones que defienden intereses sociales y colectivos, así como por la necesidad de que los más desfavorecidos puedan disponer de un mínimo de asistencia. Así, surgen de la iniciativa privada amparados por normas legales que regulan el derecho de asociación en las propias constituciones y en sus normas de desarrollo normativo.

Tras el análisis de las ONGs encontramos que las estructuras comunicativas de estas organizaciones se encuentran en el $55 \%$ de las brasileñas y en el $100 \%$ de las españolas. Son estructuras comunicativas muy limitadas en equipamiento y personal, por lo que las disponibilidades presupuestarias son escasas. En este sentido, las principales funciones son las relaciones con los medios de comunicación, con el objetivo de poder difundir sus demandas a la colectividad y a los poderes públicos. Asimismo, su actividad supone la elaboración de material periodístico como son comunicados de prensa y la organización de ruedas de prensa.

A pesar de esas limitaciones personales, materiales y presupuestarias, su presencia en las principales redes sociales es incuestionable, ya que el $100 \%$ de las ONGs de los dos países tienen presencia en Facebook, mientras que los encontramos en un 90\% (España) y un 60\% (Brasil) en Twitter. Eso supone una muy adecuada adaptación a los entornos digitales y a las nuevas técnicas digitales de comunicación. Como consecuencia de esa presencia digital, el 90\% de las ONGs españolas disponen de un community manager, frente al $50 \%$ de las ONGs brasileñas.

Por último, señalamos que los principales objetivos comunicativos en internet son la difusión de la información y de sus actividades, por lo que mantienen un marcado carácter unidireccional, soslayando así la capacidad interactiva de las redes sociales.

\section{REFERÊNCIAS}

ABONG. Associação Brasileira de Organizações Não-Governamentais. Pesquisa Fasfil 2010. Disponible en: <http://www. abong.org.br/ongs.php?id=18>. Acceso: 15 oct. 2015. 
ALMANSA MARTíNEZ, Ana. Del gabinete de prensa al gabinete de comunicación: la dirección de comunicación en la actualidad. Sevilla: Comunicación Social Ediciones y Publicaciones, 2011.

AVINA, Jeffrey. The evolutionary life cycles of non-governmental development organizations. Public Administration and Development: an International Journal of Training, Research and Practice, v. 13, n. 5, p. 453-474, dic. 1993.

CASTILLO ESPARCIA, Antonio. Relaciones públicas en las organizaciones no gubernamentales. Sphera Pública, Universidad Católica San Antonio de Murcia, n. 7, 2007.

COHEN, Joshua. Procedure and substance in deliberative democracy. In: BOHMAN, James; REGH, William (Orgs). Deliberative democracy: essays on reason and politics. Cambridge, MA: Cambridge University Press, 1997.

CONSTITUIÇÃOFederal do Brasil. Disponible en: <http://www.senado.gov.br/atividade/const/con1988/CON1988_15.09.2015/ ind.asp>. Acceso: 10 sept. 2015.

DE FELICE, Massimo. Auréola digital: a crise dos pontos de vista centrais e o fim do direito exclusivo da edição das informações. In: OLIVEIRA, Ivone de Lourdes; MARCHIORI, Marlene (Orgs.). Redes sociais, comunicação, organizações. São Caetano do Sul, SP: Difusão, 2012. Editora. (Série pensamento e prática; v. 5)

ESTEVES, João Pissarra. Opinião pública e mídias sociais: deliberação nas novas redes de comunicação e interação. In: OLIVEIRA, Ivone de Lourdes; MARCHIORI, Marlene (Orgs.) Redes sociais, comunicação, organizações. São Caetano do Sul, SP: Difusão, 2012.

FERNANDES, Rubem César. In: IOSCHPE, Evelyn Berg (Org.). Terceiro setor. desenvolvimento social sustentado. Rio de Janeiro: Paz e Terra, 1995.

GARCÍA OROSA, Berta. Theoretical approach to communication in the third sector. The necessary reclassification of organizational communication. Telos:Communication and innovation Notebooks, n. 69, Madrid, Fundación Telefónica, 2006.

GOLDSTEIN, Jonah. Public interest group and public policy: the case of the Consumers Association of Canada. Canadian Journal of Political Science, v. 12, p. 138-155, 1979.

GÓMEZ GIL, Carlos. Las ONG en España: de la aparencia a la realidad. Barcelona: Los Libros de la Catarata, 2005.

GONZÁLEZ Luis, H. Evolution theme of NGDOs as sources of media. In: VARA MIGUEL, Alfonso (Coord.). Communication in crisis: the 11-to 14-M M. Proceedings of XIX International Congress of Communication. Navarra: Navarra School of Communication University of Navarra, 2006, p. 411-428.

GRANDA, Germán; GUZMÁN, Gabriel; RAMA, Ruth. Nuevas formas de cooperación para España: el potencial de las organizaciones no gubernamentales y de las empresas. Madrid: Cideal, 1987.

HASWANI, Mariângela F. Comunicação pública: bases e abrangência. São Paulo: Saraiva, 2013.

INGENHOFF, Diana; KOELLING, Martina A. The potencial of web sites as a relationship building tool for charitable fundraising NPOs. Public Relations Review, Amsterdam, Elsevier, n. 35, p. 66-73, 2009. 
KANG, Seok; NORTON, Hanna E. Nonprofit organizations's use of the world wide web: are they sufficiently fulfilling organizational goals? Public Relations Review, Amsterdam, Elsevier, n. 30, p. 279-284, 2004.

KISIL, Marcos. Organização social e desenvolvimento sustentável: projetos de base comunitária. In: IOSCHPE, Evelyn Berg (Org.). Terceiro setor. desenvolvimento social sustentado. Rio de Janeiro: Paz e Terra, 2005.

MARCUELLO, Carmen. Capital social y organizaciones no lucrativas en España. Madrid: Fundación BBVA, 2007.

OFFE, Claus. Partidos políticos y nuevos movimientos sociales. Madrid: Ed. Sistema, 1996.

ORTEGA, Marí Luz. Estrategia de educación para el desarrollo de la cooperación española. Madrid: Ministerio de Asuntos Exteriores y Cooperación, 2007.

PERUZZO, Cicilia M. Krohling. Comunicação e culturas populares. São Paulo: Interco/CNPq/Finep, 1995.

Comunicação nos movimentos populares: a participação na construção da cidadania. Petrópolis, RJ: Vozes, 1998.

PONTES, Lúcia; BAVA, Silvio Caccia. As ONGs e as políticas públicas na construção do estado democrático. Serviço Social \& Sociedade, São Paulo, Cortez, a. XVII, n. 50, abr. 1996.

PSACHAROPOULOS, George; WOODHALL, Maureen. Educación para el desarrollo: un análisis de opciones de inversión. Tecnos, Madrid: Tenos, 1987.

SAMPEDRO, Victor; JEREZ NOVARA, Ariel; LÓPEZ-REY, José. Public image and communication strategies. En: REVILLA BLANCO, Marisa (Ed.). NGOs and politics. Madrid: Istmo Editions, 2002. p. 251-281.

SALOMON, Lester. Estratégias para o fortalecimento do terceiro setor. In: IOSCHPE, Evelyn Berg (Org.). 3ํㅗ setor: desenvolvimento social sustentado. Rio de Janeiro: Paz e Terra, 2005.

SCHERER-WARREN, Ilse. Cidadania sem fronteiras: ações coletivas na era da globalização. São Paulo: Hucitec, 1999.

SEO, Hyunjin; KIM, JI Young; YANG, Sung-Un. Global activism and new media: a study of transnational NGOs' online public relations. Public Relations Review, Amsterdam, Elsevier, n. 35, p. 123-126, 2009.

SIKIRIT CERDÁ, Soraya. Las Ongs como agentes de la cooperación y la ayuda humanitaria. UAB, Barcelona: UAB, 2003.

TORO, José Bernardo. A construção do público:cidadania, democracia e participação. Rio de Janeiro: Editora Senac Rio, 2005.

WENDHAUSEN, Henrique. Comunicação e mediação das ONGs: uma leitura a partir do canal comunitário de Porto Alegre. Porto Alegre: Edipucrs, 2003.

YANG, Amei; TAYLOR, Maureen. Relationship-building by Chinese NGOs'websites: education, notactivation. Public Relation Review, Amsterdam, Elsevier, n. 36, p. 342-351, 2010. 